\title{
DIVERSIDAD DEL GÉNERO LUTICOLA (BACILLARIOPHYCEAE) EN SEDIMENTOS HOLOCENICOS DE LA PUNA JUJEÑA, ARGENTINA
}

\author{
SABRINA BUSTOS ${ }^{1}$, MARCELO R. MORALES ${ }^{1}$ y NORA I. MAIDANA ${ }^{1,2}$
}

\begin{abstract}
Summary: Diversity of the genus Luticola (Bacyllariophyceae) in Holocenic sediments of the Dry Puna of Jujuy, Argentina. Framed in our paleoenvironmental research project, we have observed a high diversity and frequency of diatoms of the genus Luticola D.G. Mann in sediments of the last 11,000 years of Barrancas locality (S 23 18' 08,7"; W 66 $05^{\circ}$ '15,2"; $3666 \mathrm{~m}$ asl). We identified ten taxa, two of them are new species -Luticula punae and $L$. juliae-, and we described 2 different forms of $L$. mollis. Two of these species are new records for Argentina and one for South America.
\end{abstract}

Key words: Diatoms, biodiversity, highlands wetlands, Holocene.

\begin{abstract}
Resumen: En el marco de las investigaciones paleoambientales que estamos desarrollando en la localidad de Barrancas ( $23^{\circ} 18^{\prime} 08,7^{\prime \prime}$ S; $66^{\circ} 05^{\prime} 15,2^{\prime \prime} \mathrm{O}$; 3666 m snm) detectamos, en sedimentos de los últimos 11.000 años, una alta diversidad y frecuencia de representantes del genero Luticola D.G. Mann. Se identificaron 10 especies, de las cuales dos son nuevas (Luticola punae sp. nov. y Luticola juliae sp. nov.). Se describen dos morfotipos de Luticola mollis. Dos de estas especies se citan por primera vez para Argentina y una para Sudamérica.
\end{abstract}

Palabras claves: Diatomeas, biodiversidad, humedales de altura, Holoceno.

\section{INTRODUCCIÓN}

Las especies de Luticola D.G. Mann se encuentran en ambientes aerófilos o sub-aéreos, como musgos terrestres semi-húmedos o en rocas permanentemente húmedas (Van de Vijver \& Mataloni, 2008). El género ha sido detectado en diversas áreas del planeta, incluidos algunos de los hábitats más extremos como la Antártida y varias islas del Atlántico Sur. Al respecto, Van de Vijver et al. (2011) sostienen que es uno de los géneros dominantes en hábitats terrestres de la región Antártica. Por su parte, Rumrich et al. (2000) registran que en los Andes, desde Venezuela hasta

1 IBBEA (CONICET - UBA); Laboratorio de Diatomeas Continentales. Lab. 15, Pabellón 2, piso 4, Ciudad Universitaria. Int. Güiraldes 2160 (C1428EGA) C.A.B.A. E-mail: sabrina.bustos.m@gmail.com 2 DBBE - FCEyN - UBA. la Patagonia, existen especies de este género en diversos ambientes que van desde los $800 \mathrm{~m}$ snm hasta los Altos Andes, a más de $4300 \mathrm{~m}$ snm, siendo particularmente notable su presencia en el norte de Chile, por encima de los $4000 \mathrm{~m}$ snm.

De acuerdo con Round et al. (1990) y Levkov et al. (2013), el género incluye células naviculoides solitarias o que raramente forman cadenas. Sus valvas son planas, lineales, lanceoladas o elípticas, con extremos desde no proyectados hasta capitados. Los rasgos distintivos del género son la presencia de un solo estigma, estrías uniseriadas formadas por areolas circulares a elípticas, manto con una única hilera de areolas, nódulo central fuertemente engrosado y presencia de un canal marginal ocluido internamente por hímenes a lo largo de la unión entre el manto y la superficie valvar. El esternón es bastante angosto, expandido y engrosado en el centro formando un estauro corto, perforado en uno de sus lados por el estigma. Los extremos distales del rafe pueden ser casi rectos o curvados, 
hasta incluso tener forma de gancho; cuando están curvados, usualmente es en el mismo sentido que los extremos proximales. Estos últimos están más o menos curvados en sentido opuesto al estigma. Las areolas están ocluidas internamente por hímenes que confluyen formando una banda transapical. El estigma se abre hacia el interior por una hendidura labiada y hacia el exterior por un poro simple.

En los últimos años, algunos autores (Van de Vijver \& Mataloni, 2008; Van de Vijver et al., 2011; Esposito et al., 2008; Kopalová et al., 2009; Zidarova et al., 2014; Glushchenko \& Kulikovskiy, 2015) comenzaron a revisar la diversidad taxonómica del género y publicaron varias nuevas especies.

Recientemente Levkov et al. (2013) publicaron una revisión del género Luticola abarcando especies de todos los continentes y zonas climáticas aunque con énfasis en América Central, Sudamérica, Antártida e Islas del Atlántico Sur. En su monografía presentan 200 taxones, de los cuales 92 son nuevas especies. Asimismo, incluyeron nueva información sobre distribución geográfica y preferencias ecológicas para cada taxón y describieron un nuevo género (Luticolopsis Levkov op cit.).

Se cuenta con escasa información sobre la flora de diatomeas de ambientes de altura en Sudamérica en general y en la zona de la puna seca y de la provincia de Jujuy en particular (Maidana \& Seeligman, 2006; Maidana et al., 1998, 2009, 2011; Seeligman \& Maidana, 2003; Seeligmann et al., 2008) por lo que resulta importante realizar estudios de taxonomía y diversidad que ayuden a completar y mejorar los datos existentes.

Los especímenes de Luticola estudiados en este trabajo fueron observados en sedimentos muestreados para los estudios paleoambientales que nuestro grupo de investigación viene realizando en la localidad Barrancas (Depto. Cochinoca, Jujuy) (Morales et al., 2014, 2015; Pirola et al., 2015). Estos estudios se orientan a generar información paleoecológica y paleoambiental relevante para comprender la dinámica de las ocupaciones humanas detectadas en esta área por el proyecto arqueológico marco de esta investigación (PICT 2014-2863). Así, este estudio de taxones infragenéricos de Luticola presentes en la localidad de Barrancas tiene alcances multidisciplinarios ya que, al precisar la identificación taxonómica de las diatomeas altoandinas, además de ampliar nuestro conocimiento de su diversidad, nos permite ajustar las interpretaciones paleoambientales.

\section{Materiales y Métodos}

La Puna Argentina es el sector del territorio nacional ubicado por encima de los $3000 \mathrm{~m}$ snm en los Andes Tropicales. Constituye una gran cuenca endorreica que puede ser subdividida en cuencas menores interconectadas que drenan en distintos humedales: lagunas someras de agua dulce o salobre, salares, salinas y vegas o ciénagas. La característica predominante de la Puna es su aridez, con precipitaciones estacionales que van desde más de $400 \mathrm{~mm} /$ año en el sector noreste de la Puna Seca de Jujuy hasta menos de $50 \mathrm{~mm} / \mathrm{año}$ en el sector sudoeste de la Puna Salada catamarqueña. En términos climáticos, Garreaud et al. (2003, 2009) han establecido que las precipitaciones en los Andes Tropicales -y consecuentemente en la Puna- están fuertemente relacionadas a la circulación atmosférica de alto nivel -i.e. al balance entre los vientos del este (Alisios) y los vientos del oeste (Westerlies)- siendo el Amazonas la fuente de la humedad que ingresa en el territorio entre diciembre y marzo debido al sistema Monzónico Sudamericano (Zhou \& Lau, 1998).

Elárea de estudio, usualmente llamada Barrancas, se encuentra ubicada en las inmediaciones del pueblo de Abdón Castro Tolay en la Puna Desértica, que es la zona de transición entre la Puna Seca y la Salada, a ca. $3500 \mathrm{~m} \mathrm{snm}$ y a $15 \mathrm{~km}$ al oeste de Salinas Grandes, en el departamento de Cochinoca, Provincia de Jujuy. Las precipitaciones en esta localidad alcanzan los $180 \mathrm{~mm} / \mathrm{año}$, concentradas en los meses estivales siguiendo el patrón regional descripto anteriormente.

Los muestreos para los estudios paleoambientales que son marco de esta investigación se realizaron en la porción media del río Barrancas ( $23^{\circ} 18^{\prime} 08,7^{\prime}$ ' S; 66 05' 15,2" O; $3666 \mathrm{~m} \mathrm{snm}$ ), uno de los cursos tributarios permanentes del sector inferior del río Las Burras (cuenca Salinas Grandes-Guayatayoc) (Fig. 1). El material estudiado procede de 62 muestras: 29 provenientes de un testigo sedimentario (TC1) de 2,30 $\mathrm{m}$ de profundidad, perforado en la planicie de inundación del río y cuyos sedimentos corresponden al lapso comprendido entre los 11.000 y $4500 \mathrm{AP}$ aproximadamente y 33 muestras obtenidas del 


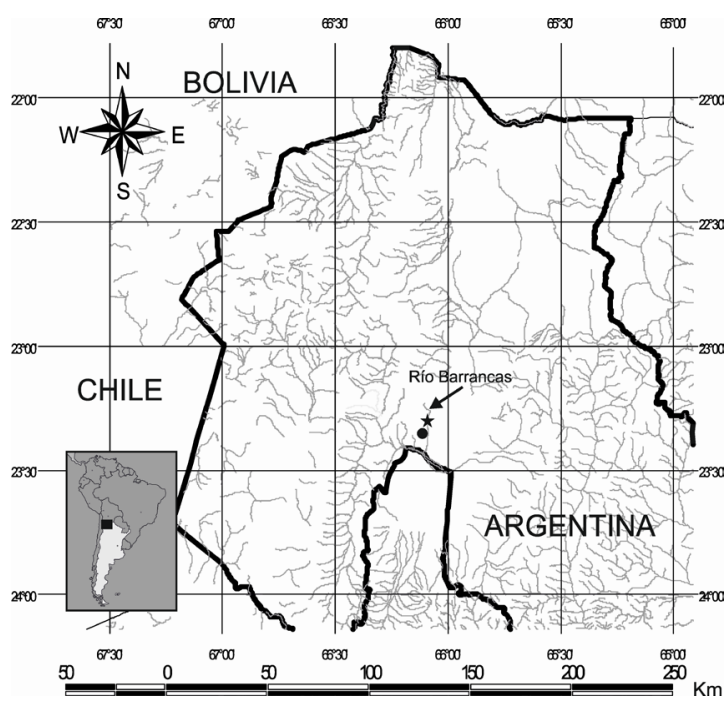

Fig. 1. Ubicación del área de muestreo. • Localidad de Abdón Castro Tolay. $\star$ Área de muestreo

segmento inferior (primeros $3,30 \mathrm{~m}$ ) del perfil PCc2, de 7,40 m de longitud, extraído de una terraza fluvial ubicada a menos de $20 \mathrm{~m}$ de TC1 y que representa un lapso que va de los 4500 a los 2000 años AP aproximadamente, según las dataciones radiocarbónicas disponibles (datos inéditos).

El tratamiento del material se hizo siguiendo los procedimientos estándar sugeridos por Battarbee (1986): oxidación de la materia orgánica con $\mathrm{H}_{2} \mathrm{O}_{2}$ $(30 \%)$ a $80{ }^{\circ} \mathrm{C}$, eliminación de carbonatos con $\mathrm{HCl}$ $10 \%$ y neutralización de la suspensión resultante mediante lavados sucesivos con agua destilada. El material tratado fue montado en portaobjetos de manera permanente con Naphrax ${ }^{\circledR}$. Las valvas de Luticola se observaron con un microscopio binocular (MO) Reichert-Jung Polyvar a 1000x y las fotografías fueron obtenidas con una cámara digital Cannon EOS 600D adaptada al microscopio. La microscopía electrónica de barrido se realizó en un Zeiss Supra40, previo metalizado con oro, en el Centro de Microscopías Avanzadas de la Facultad de Ciencias Exactas y Naturales de la Universidad de Buenos Aires. El material estudiado se encuentra depositado en la colección del Laboratorio de Diatomeas Continentales del DBBE, FCEyN, UBA.

Las principales variables morfométricas de los especímenes -largo, ancho, estrías c/10 $\mu \mathrm{m}$ y areolas $\mathrm{c} / 10 \mu \mathrm{m}$ - fueron medidas con el programa Zeiss AxioVision 40 v. 4.8.2.0 sobre fotografías digitales obtenidas con MO y MEB. Los valores morfométricos especificados en las descripciones corresponden a las medidas obtenidas en este trabajo. Los análisis estadísticos se realizaron con el software PAST v. 3.11 (Hammer et al., 2001).

Las descripciones se hicieron empleando la terminología utilizada por Levkov et al. (2013), Krammer \& Lange-Bertalot (1986, 1988, 1991a, 1991b), Round et al. (1990), Rumrich et al. (2000) y trabajos puntuales que contienen especies de este género, como los de Van de Vijver et al. (2002a, 2002b, 2006, 2011), Metzeltin et al. (2005), Metzeltin \& Lange-Bertalot (2007), Van de Vijver \& Mataloni (2008), Esposito et al. (2008), Kopalová et al. (2009), Zidarova et al. (2014) y Glushchenko \& Kulikovskiy (2015).

\section{Resultados y Discusión}

Se presentan las descripciones de las especies identificadas durante el estudio del testigo y perfil de la localidad de Barrancas, algunas de las cuales son nuevas citas para Argentina y otras para Sudamérica. Al final de las descripciones, hemos resumido en la Tabla 1 los principales caracteres diagnósticos para la identificación de las distintas especies encontradas.

Luticola incana Levkov et al. Figs. 2: A-D

Levkov et al. (2013): 135; Figs. 26: 55-60; 32: 52-60

Valvas lineales a lineal-elípticas con márgenes variablemente convexos. Ápices redondeados a truncados. Área axial angosta y lineal; área central ligeramente asimétrica, transversalmente rectangular a cuneada, bordeada en cada margen por 3-4 areolas. Un solo estigma presente en el área central, más próximo al margen que al centro. Rafe con las fisuras externas, proximales y distales, cortas, ligeramente curvadas hacia el lado opuesto del estigma. Estrías transapicales radiales cerca del centro de la valva, volviéndose fuertemente radiadas hacia los ápices; notablemente areoladas, compuestas por 3-4 areolas desde redondeadas hasta transapicalmente alargadas.

Dimensiones: eje apical 11,0-24,5 $\mu \mathrm{m}(\mathrm{N}=15)$, eje transapical 4,5-8,5 $\mu \mathrm{m}(\mathrm{N}=15)$; estrías 16-21 en $10 \mu \mathrm{m}(\mathrm{N}=11)$. 


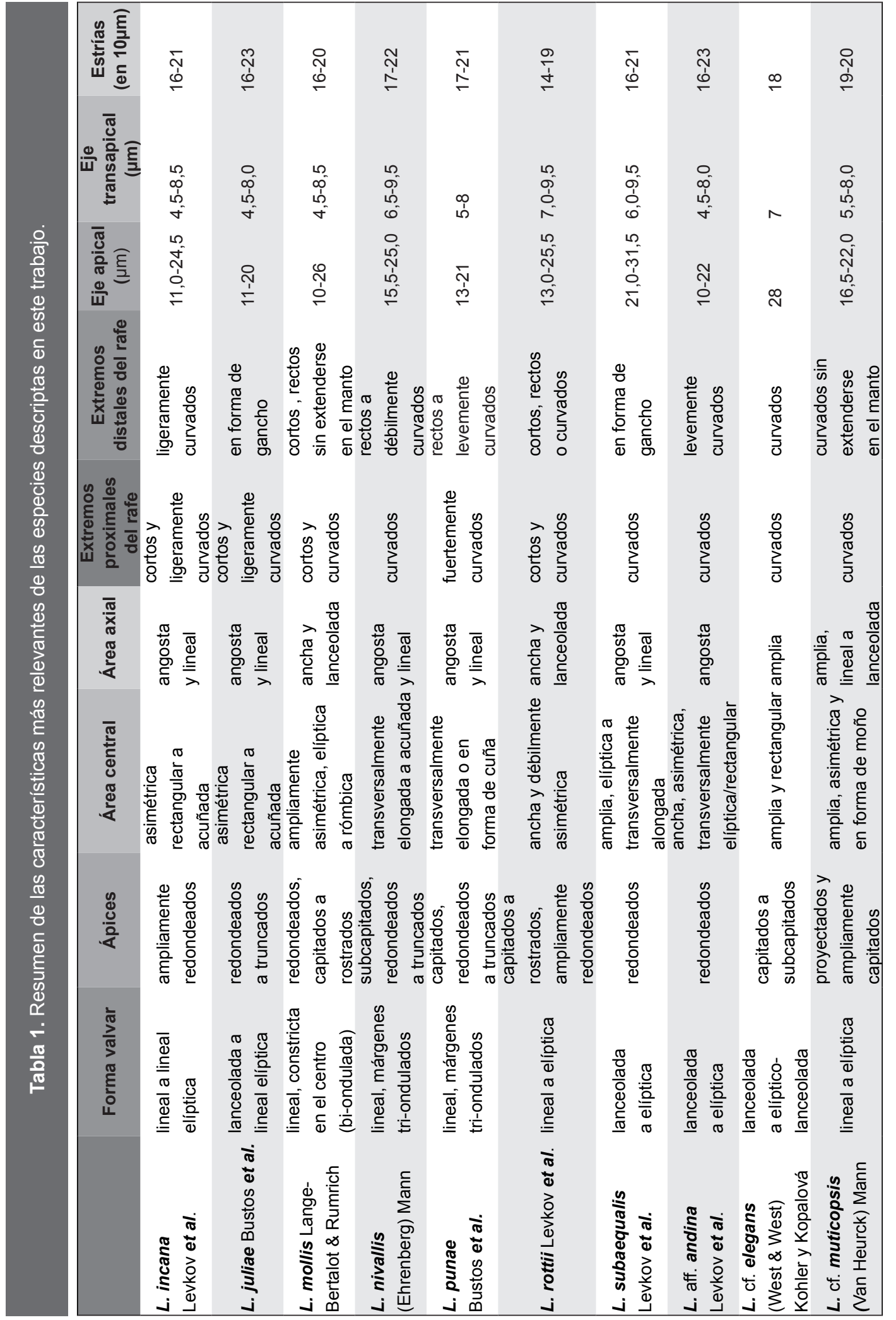


S. Bustos et al. - Diversidad del género Luticola en la Puna Jujeña
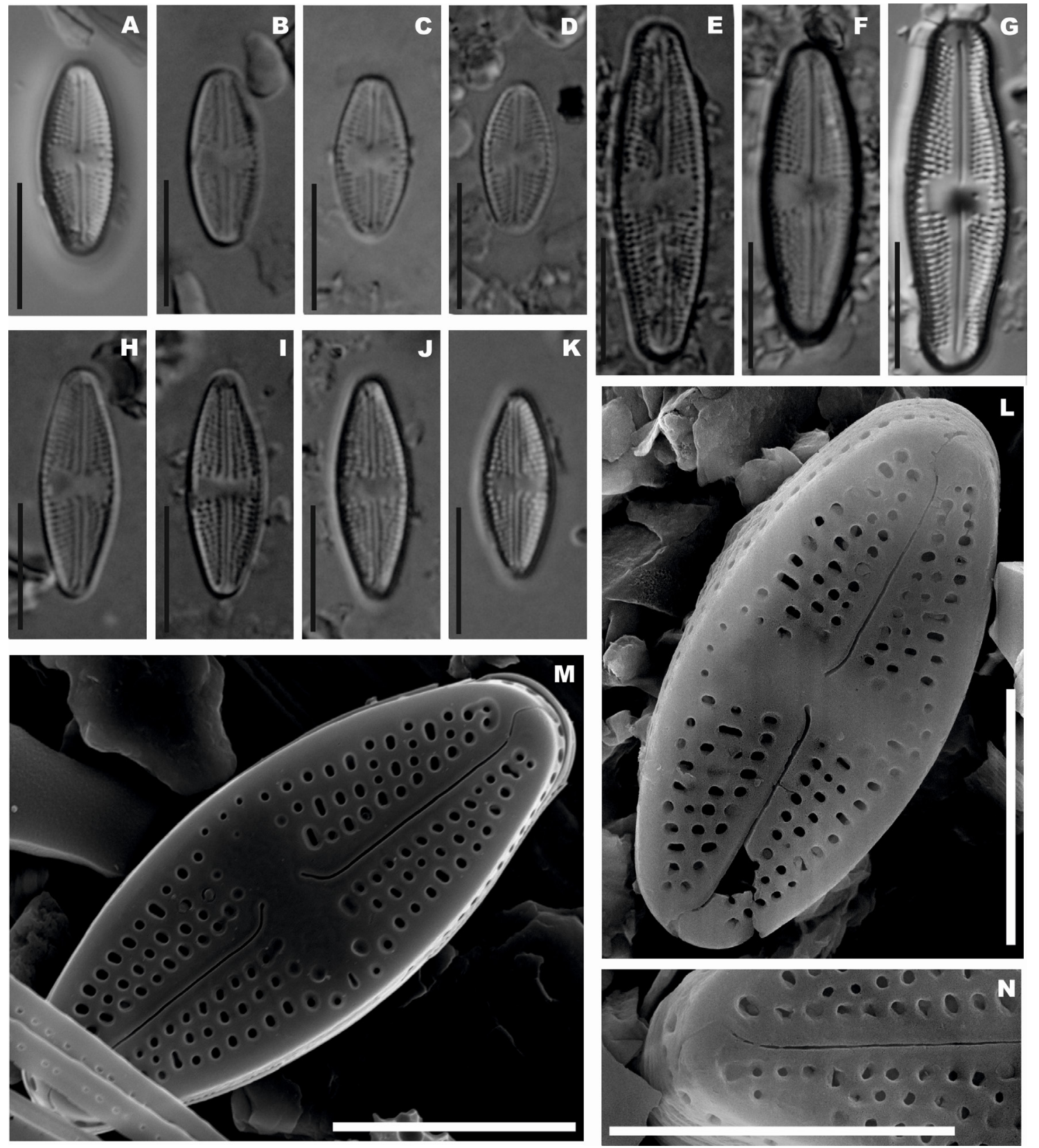

Fig. 2. A-D: Luticola incana (MO); E-F: L. subaequalis (MO); G: L. cf. elegans (MO); H-N: L. juliae nov. spec. $\mathrm{H}-\mathrm{K}$ (MO, corresponden al holotipo); L-M (MEB) vista externa; N (MEB) detalle del extremo de una valva, nótese la curvatura del extremo distal del rafe. Escala negra $=10 \mu \mathrm{m}$ (Figs. A-K); escala blanca $=5 \mu \mathrm{m}$ (Figs. L-N).

Distribución geográfica: Perú y dos localidades en Chile (Levkov et al., 2013). Primera cita para Argentina.

Material observado: TC1: muestras 17, 25, 97, 105, 161, 225; PCc2: muestras 7, 9, 10 y 12.
Luticola juliae Bustos, S., M. R. Morales \& N. Maidana nov. spec. Figs. 2: H-N

Valvas lanceoladas a elíptico-lanceoladas con ápices cuneado-redondeados. Área axial angosta y 
lineal; área central angosta, asimétrica, con forma de cuña o transversalmente rectangular, bordeada en cada margen por 2-3 areolas. El estigma solitario y elongado presente cerca del margen valvar, por esta razón es difícil de reconocer en el MO. Fisuras proximales del rafe cortas, curvadas hacia el lado opuesto del estigma. Fisuras distales en forma de gancho, curvadas hacia el lado del estigma, extendidas sobre el manto. Estrías transapicales levemente radiales, compuestas por 3-4 areolas redondeadas a levemente alargadas en sentido transapical.

Diagnosis: Valves lanceolate to elliptic-lanceolate, with rounded or wedge-shaped apices. Axial area linear and narrow; central area narrow and asymmetrical, transversally rectangular or wedgeshaped, bordered on each margin by 2-3 areolae. Single elongated stigma located close to the valve margin. Proximal raphe endings short and curved towards the side opposite to the stigma. Distal raphe endings hooked, curved towards the side of the stigma and extended onto the mantle. Transapical striae slightly radiate, composed of 3-4 rounded to slightly elongated areolae.

Dimensiones: eje apical 11-20 $\mu \mathrm{m}(\mathrm{N}=53)$, eje transapical 4,5-8,0 $\mu \mathrm{m}(\mathrm{N}=53)$; estrías $16-23$ en 10 $\mu \mathrm{m}(\mathrm{N}=46)$.

Holotipo: ARGENTINA. Prov. Jujuy: Dpto. Cochinoca, Abdón Castro Tolay Morales M. R. Marzo de 2013 (BA) BA 49537 Herbario de Plantas Celulares, Museo Argentino de Ciencias Naturales "Bernardino Rivadavia" (preparado microscópico y material procesado).

Localidad tipo: Barrancas (Depto. Cochinoca, Jujuy, Argentina), muestra TC1 M225.

Material observado: TC1: muestras 1, 9, 25, 41, 65, 73, 89, 105, 129, 161, 177, 193, 217, 225; PCc2: muestras 7, 9, 10, 11 y 12 .

Observaciones: Luticola juliae puede distinguirse de L. incana por su contorno valvar más lanceolado, las fisuras externas distales en forma de azada (Fig. 2-I) en lugar de curvadas y cortas y el estigma ubicado próximo al margen, con la abertura externa más elongada. Se diferencia de L. andina porque esta tiene ápices redondeados, área central elíptica y las fisuras distales externas rectas y cortas y de $L$. rotunda por la forma general del contorno valvar y del área axial, que en esta especie es amplia y lanceolada.
Lamentablemente, las descripciones de $L$. andina y $L$. incana no están apoyadas por imágenes de MEB y en el caso de $L$. rotunda solo hay una ilustración de una vista externa en MEB (Levkov et al., 2013).

Luticola mollis Lange-Bertalot \& Rumrich Figs.3: A-J y 4: A-J

Rumrich et al. (2000): 149: Figs. 61: 5-6

Levkov et al. (2013): 164: Figs. 200: 1-17, 36

Valvas lineales con extremos desde rostrados hasta capitados y ápices anchamente redondeados. Área axial expandida cerca del área central; área central asimétrica, de forma variable, generalmente cuneada y limitada cerca de cada margen por 3-4 areolas redondeadas. Estigma ubicado en el área central, equidistante entre el centro de la valva y el margen, con la abertura externa pequeña y redondeada. Fisuras proximales externas del rafe cortas, levemente curvadas hacia el lado opuesto del estigma y las distales cortas, rectas, algo alejadas de los extremos. Estrías transapicales radiales, compuestas por 2-3 areolas elongadas transapicalmente, generalmente de mayor longitud cerca del margen valvar.

Dimensiones: eje apical 10-26 $\mu \mathrm{m}(\mathrm{N}=109)$, eje transapical en la constricción 4,5-8,5 $\mu \mathrm{m}(\mathrm{N}=109)$; estrías $16-20$ en $10 \mu \mathrm{m}(\mathrm{N}=109)$.

Distribución geográfica: Parque Nacional Lauca, Chile (Rumrich et al., 2000), Jujuy y Catamarca, Argentina (Maidana \& Seeligman, 2006; Seeligman et al., 2008; Maidana et al., 2009).

Observaciones: Hemos hallado algunos especímenes cuyas dimensiones exceden las indicadas por Rumrich et al. (2000) (eje apical 18-30 $\mu \mathrm{m}$; eje transapical, en la constricción, 9-11 $\mu \mathrm{m} ; 17-19$ estrías en $10 \mu \mathrm{m})$. Dado que las principales características morfológicas coinciden con las de la especie, en este trabajo ampliamos su rango de variación.

En los ejemplares analizados se observó un grado variable de asimetría respecto del eje apical y en la forma general del contorno de las valvas, que nos ha permitido individualizar dos morfotipos. El morfotipo I (Fig. 3: A-J, ver Rumrich et al. (2000), p. 149 Fig. 61: 6 y Levkov et al. (2013), p. 164, fig. 200: 2-11, 13$16,36)$ tiene ambos márgenes bi-ondulados aunque del lado del estigma la ondulación es mucho más marcada. El morfotipo II (Fig. 4: A-J ver Rumrich et al. (2000), p. 149 fig. 61: 6; Levkov et al. (2013), p. 164, fig. 200: 1, 12, 17) es marcadamente 
S. Bustos et al. - Diversidad del género Luticola en la Puna Jujeña
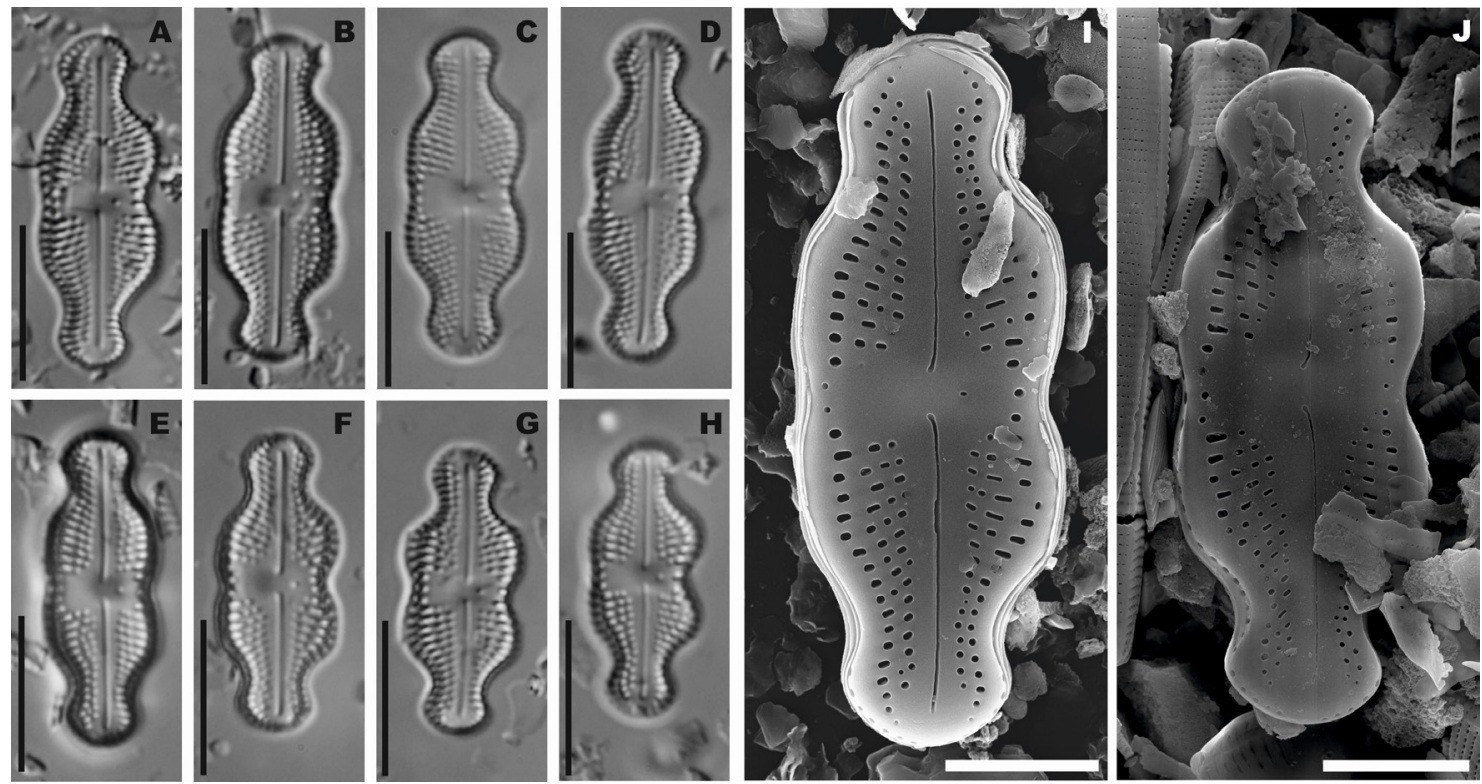

Fig. 3. Luticola mollis morfotipo I. A-H (MO); I-J (MEB) vistas externas. Escala negra $=10 \mu \mathrm{m}$ (Figs. A-H); escala blanca $=5 \mu \mathrm{m}$ (Figs. I-J).
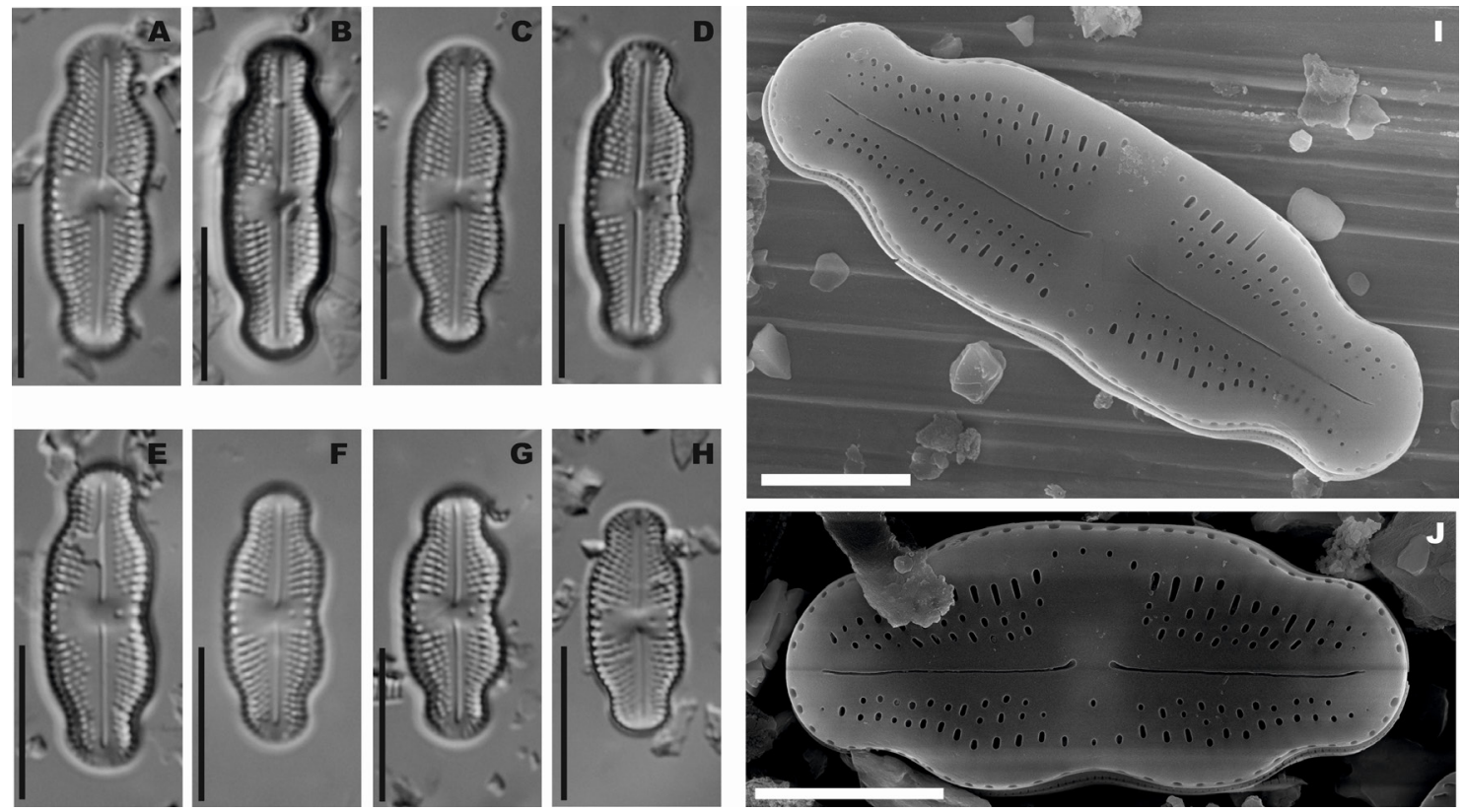

Fig. 4. Luticola mollis morfotipo II. A-H (MO); I-J (MEB) vistas externas. Escala negra $=10 \mu \mathrm{m}$ (Figs. A-H); escala blanca $=5 \mu \mathrm{m}$ (Figs. I-J). 
asimétrico, con una ondulación muy reducida o incluso ausente en la hemivalva opuesta al estigma. Los análisis estadísticos (ANOVA) realizados con las características métricas de 44 valvas del morfotipo I y 63 del morfotipo II solo mostraron diferencias significativas en cuanto al ancho $\left(\mathrm{p}=6,368 \mathrm{e}^{-8}\right)$. Los resultados restantes del ANOVA indicaron que no hay diferencias significativas en cuanto a la longitud $(\mathrm{p}=0,081)$ y a la relación longitud-ancho $(\mathrm{p}=0,08733)$.

Material observado: TC1: muestras 1, 17, 25, 33, 105, 113, 129; PCc2: muestra 11.

Luticola nivalis (Ehrenberg) D.G. Mann Figs. 5: F-H

Rumrich et al. (2000): 362: Fig.61: 7

Levkov et al. (2013): 175: Figs. 177: 1-40

Valvas lineales con márgenes triondulados. Extremos rostrados a capitados, con ápices, redondeados o truncados. Área axial lineal, ligeramente expandida cerca del área central, que es ancha, transversalmente elongada hasta cuneada, bordeada por una fila de 3-4 areolas en cada margen. Estigma solitario en el área central, equidistante del centro de la valva y del margen. Ramas del rafe rectas a levemente curvadas. Fisuras proximales externas curvadas en el sentido opuesto al estigma. Fisuras distales en forma de gancho, primero débilmente curvadas hacia el mismo lado que los extremos proximales y luego curvados hacia el lado opuesto, no extendidas en el manto. Estrías transapicales fuertemente radiales, compuestas por 3-4 filas de areolas transversalmente elongadas.

Dimensiones: eje apical 15,5-25,0 $\mu \mathrm{m}(\mathrm{N}=12)$, eje transapical 6,5-9,5 $\mu \mathrm{m}(\mathrm{N}=12)$; estrías $17-22$ en 10 $\mu \mathrm{m}(\mathrm{N}=11)$.

Distribución geográfica: reportada para todos los continentes; ampliamente distribuida en agua dulce (Levkov et al., 2013). Provincias de Jujuy y Catamarca, Argentina (Maidana \& Seeligman, 2006; Seeligman et al., 2008; Maidana et al., 2009).

Observaciones: algunos de los ejemplares de esta localidad exceden ligeramente las dimensiones indicadas por Levkov et al. (2013) (eje apical 11-21 $\mu \mathrm{m}$; eje transapical 6-8 $\mu \mathrm{m})$.

Material observado: TC1: muestras: 1, 9, 65, 73, 121, 129, 177, 193, 225; PCc2 muestras 7 y 12.
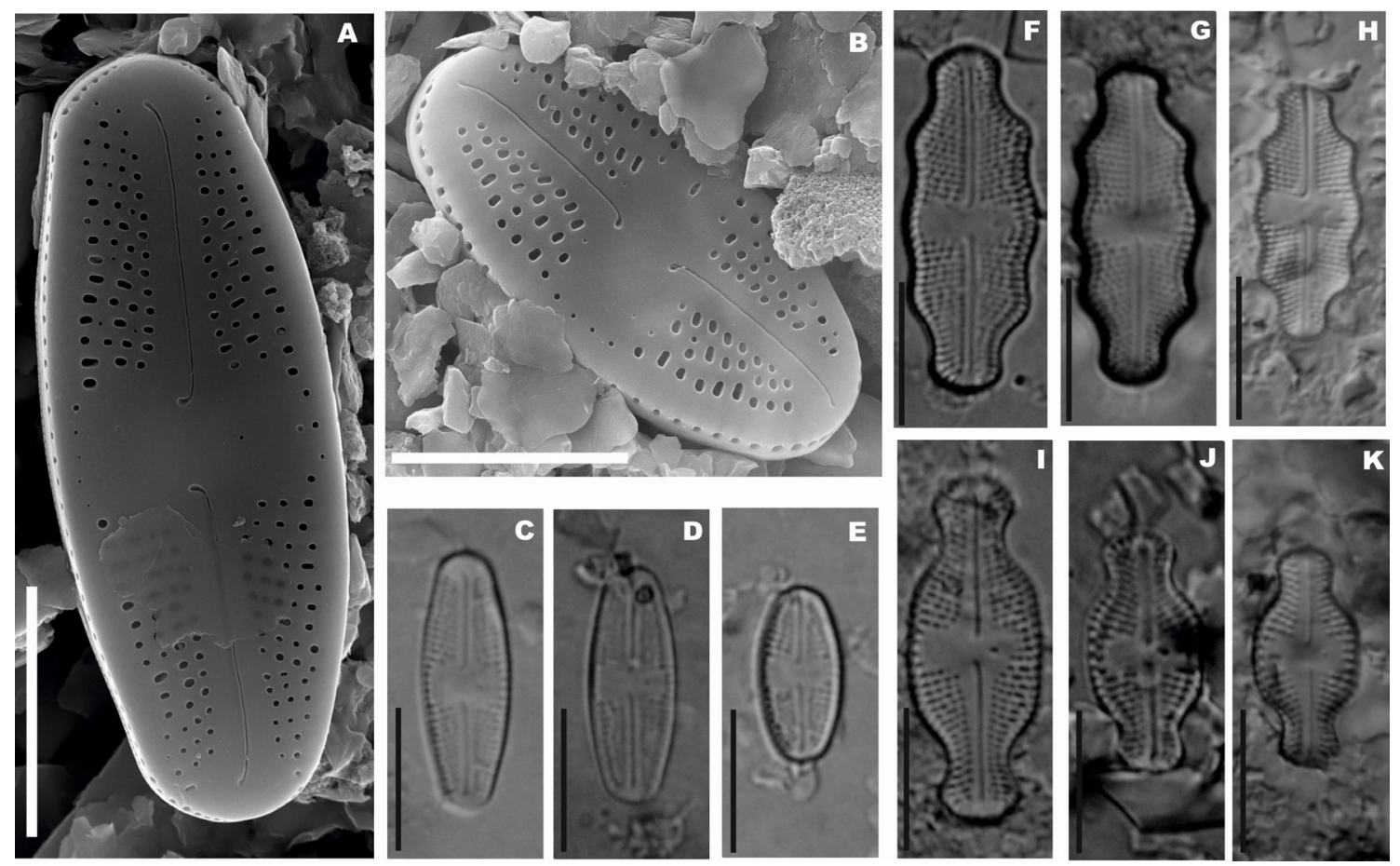

Fig. 5. A-E: Luticola aff. andina A, B (MEB) vistas externas; C-E (MO). F-H: L. nivalis (MO). I-K: L. rottii (MO). Escala negra $=10 \mu \mathrm{m}$ (Figs. C-K); escala blanca $=5 \mu \mathrm{m}$ (Figs. A, B). 


\section{S. Bustos et al. - Diversidad del género Luticola en la Puna Jujeña}

Luticola punae Bustos, S., M. R. Morales \& N. Maidana nov. spec. Figs. 6: D-H

Valvas lineales con márgenes tri-ondulados, simétricos y extremos capitados. Área axial amplia y lineal; área central transversalmente elongada o en forma de cuña, bordeada en cada margen por 3-4 areolas. Estigma solitario, ubicado en el área central aproximadamente equidistante del centro de la valva y del margen. Ramas del rafe rectas. Fisuras proximales externas fuertemente curvadas. Fisuras distales rectas o levemente curvadas, no extendidas sobre el manto. Las fisuras proximales y distales externas del rafe se curvan hacia el lado opuesto al del estigma. Estrías transapicales moderadamente radiales y notablemente areoladas aun al MO, compuestas por 3-4 areolas redondeadas en el centro a transversalmente elongadas hacia el borde de la valva.

Diagnosis: Valves linear with tri-undulate and symmetric margins, and capitate apices. Axial area broad and linear; central area transversally elongated or wedge-shaped, bordered on each margin by 3-4 areolae. Single rounded stigma located halfway between valve center and margin. Raphe branches straight. Proximal external raphe endings strongly curved. Distal raphe endings straight or slightly curved, not extended onto the mantle. Distal and proximal external raphe endings are curved towards the side opposite to the stigma. Transapical striae moderately radiate and evidently areolated even with LM, composed by 3-4 rounded areolae at the center of the valve and rounded to elongated towards the valve margin.

Dimensiones: eje apical 13-21 $\mu \mathrm{m}(\mathrm{N}=9)$, eje transapical 5-8 $\mu \mathrm{m}(\mathrm{N}=9)$; estrías 17-21 en $10 \mu \mathrm{m}$ $(\mathrm{N}=4)$.

Holotipo: ARGENTINA. Prov. Jujuy: Dpto. Cochinoca, Abdón Castro Tolay Morales M. R. marzo de 2013 (BA) 49536 Herbario de Plantas Celulares, Museo Argentino de Ciencias Naturales "Bernardino Rivadavia (un preparado microscópico y material procesado).

Localidad tipo: Barrancas, muestra TC1 M129.

Observaciones: Los ejemplares identificados guardan cierta semejanza, principalmente en cuanto al contorno valvar, con $L$. nivalis, $L$.
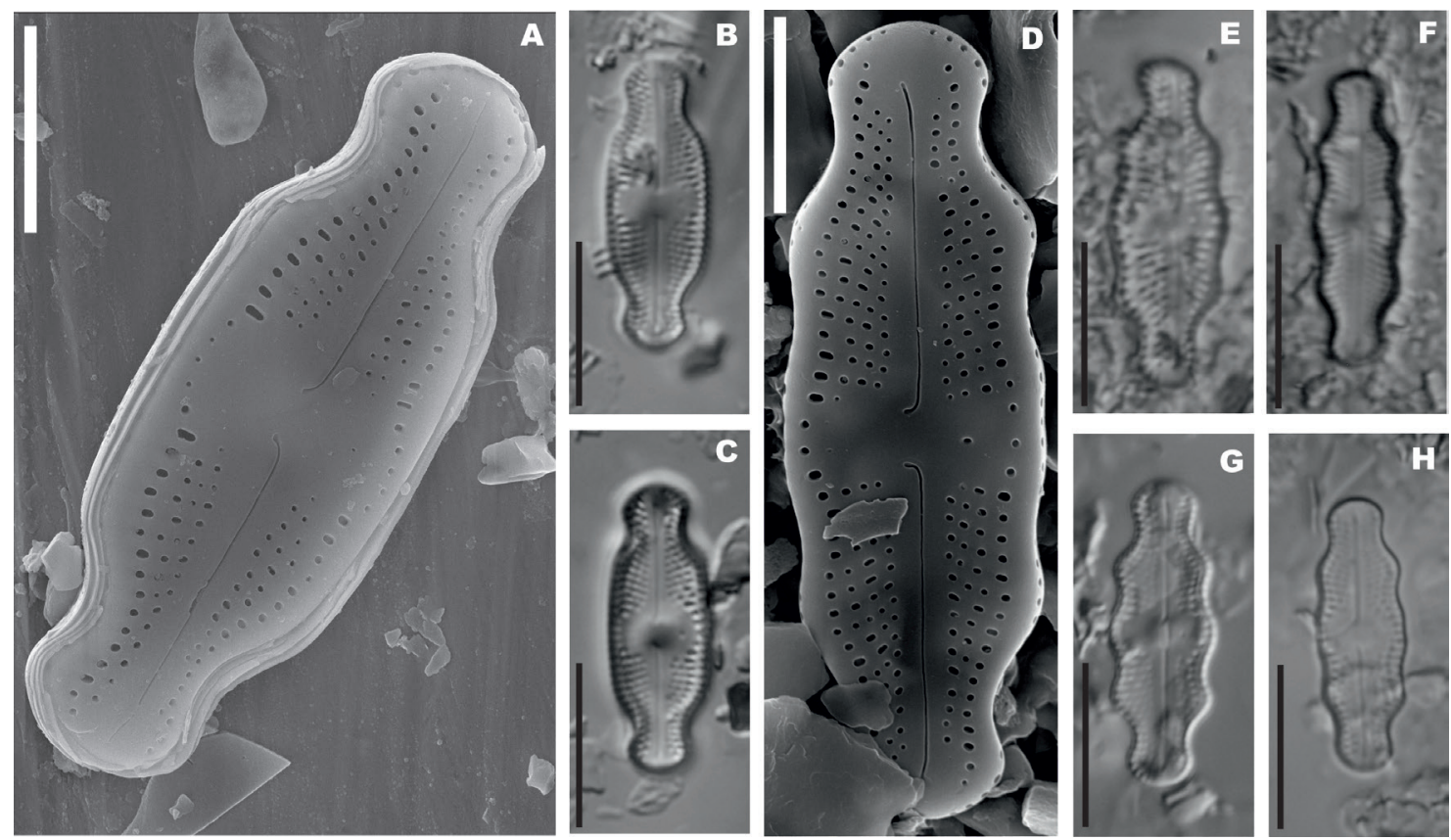

Fig. 6. A-C: Luticola cf. muticopsis A (MEB) vista externa; B-C (MO). D-H: L. punae. D (MEB) vista externa; $\mathrm{E}-\mathrm{H}(\mathrm{MO}), \mathrm{F}-\mathrm{G}$ (holotipo). Escala negra $=10 \mu \mathrm{m}$ (Figs. B, C, E-H); escala blanca $=5 \mu \mathrm{m}$ (Figs. A, D). 
pulchra, L. quinquenoides, L. undulata y $L$. triundulata. Las dos primeras especies tienen los extremos distales del rafe en forma de gancho. $L$. pulchra, además, posee mayor densidad de estrías. L. quinquenoides también presenta una mayor densidad de estrías, el eje transapical es más largo $\mathrm{y}$, a diferencia de L. punae, las fisuras distales del rafe tienen forma de gancho y se extienden sobre el manto valvar. L. undulata se diferencia de $L$. punae por tener los extremos distales del rafe en forma de gancho, extendidos hasta el manto y en la presencia de areolas fantasma en el centro de la valva. Finalmente, $L$. triundulata tiene un número menor de areolas por estría (2-3) y una fascia longitudinal hialina que interrumpe las estrías.

Material observado: TC1: muestras 113, 129 у 225 .

\section{Luticola rottii Levkov et al. Figs. 5: I- K}

Levkov et al. (2013): 211: Figs. 139: 1-25

Valvas casi rectangulares, con márgenes suavemente convexos a casi paralelos en el centro de la valva. Extremos rostrados a capitados, con ápices anchamente redondeados. Área axial ancha, lanceolada, dilatada cerca del área central, la que es ancha, débilmente asimétrica, transversalmente elíptica a cuneada, bordeada a ambos lados por estrías acortadas. Estigma ubicado en el área central, cerca del centro de la valva, con el foramen externo circular. Ramas del rafe rectas, con las fisuras proximales externas cortas y curvadas hacia el lado opuesto del estigma, con los extremos ligeramente expandidos. Fisuras distales externas cortas, rectas o curvadas hacia el mismo lado que los extremos proximales. Estrías radiales cerca del centro de la valva, volviéndose fuertemente radiales hacia los ápices, notablemente areoladas. Las estrías del lado del estigma están formadas por 2-3 areolas mientras que las del lado opuesto están compuestas por una sola línea de areolas.

Dimensiones: eje apical 13,0-25,5 $\mu \mathrm{m}(\mathrm{N}=8)$, eje transapical 7,0-9,5 $\mu \mathrm{m}(\mathrm{N}=8)$; estrías 14-19 en $10 \mu \mathrm{m}(\mathrm{N}=7)$.

Distribución geográfica: hasta el momento solo reportada para el Parque Nacional Lauca, Chile (Levkov et al., 2013). Primera cita para Argentina. Material observado: TC1: muestras 97, 105, 121, 129, 145, 177, 225; PCc2: muestras 7 y 9.
Luticola subaequalis Levkov et al. Figs. 2: E-F

Levkov et al. (2013): 227: Figs. 13: 32-44; 15: 4; 25: 19-37

Valvas lanceoladas a elíptico-lanceoladas con extremos subrostrados o no proyectados y ápices redondeados. Área axial angosta y lineal; área central amplia, transversalmente elíptica, bordeada en cada margen por 3-5 areolas aisladas. Estigma con el orificio externo transversalmente elíptico algo desplazado hacia el margen. Las ramas del rafe son rectas, con las fisuras proximales curvadas en sentido opuesto al estigma y las distales en forma de gancho, curvadas primero en el mismo sentido que las proximales y luego en sentido opuesto. Estrías transapicales radiales a lo largo de toda la valva, notablemente areoladas, compuestas por 4-5 areolas redondeadas.

Dimensiones: eje apical 21,0-31,5 $\mu \mathrm{m}(\mathrm{N}=13)$, eje transapical 6,0-9,5 $\mu \mathrm{m}(\mathrm{N}=13)$; estrías 16-21 en $10 \mu \mathrm{m}(\mathrm{N}=13)$.

Distribución geográfica: reportada para varias localidades en Macedonia, Suroeste de China y Turquía (Levkov et al., 2013). Primera cita para América del Sur.

Material observado: TC1: muestras 1, 41, 57, 129, 177, 217; PCc2: muestras 7, 9, 10 y 12.

Luticola aff. andina Levkov et al. Figs. 5: A-E

Levkov et al. (2013): 64: Figs. 26: 1-30

Valvas lineal-lanceoladas a elípticas en los especímenes de mayor tamaño y lineal-elípticas a elípticas en los más pequeños. Extremos no proyectados, con ápices generalmente redondeados. Área axial amplia, lanceolada, algo expandida cerca del área central, la que es ancha, asimétrica, transversalmente elíptica a rectangular y limitada a cada lado por una hilera de 3-4 areolas. Un estigma en el área central, más cerca del margen valvar que del nódulo central. Rafe casi recto, con las fisuras proximales externas cortas, ligeramente curvadas hacia el lado opuesto al estigma y las distales cortas y ligeramente curvadas, también hacia el lado opuesto del estigma, no extendidas en el manto de la valva. Estrías transapicales moderadamente radiales formadas por 2-4 areolas ligeramente elongadas en sentido transversal. Areolas de forma variable aún dentro de una misma estría.

Dimensiones: eje apical 10-22 $\mu \mathrm{m}(\mathrm{N}=73)$, eje 


\section{S. Bustos et al. - Diversidad del género Luticola en la Puna Jujeña}

transapical 4,5-8,0 $\mu \mathrm{m}(\mathrm{N}=72)$; estrías $16-23$ en 10 $\mu \mathrm{m}(\mathrm{N}=65)$.

Distribución geográfica: En algunas localidades en el Parque Nacional Lauca, Prov. Parinacota, Chile (Levkov et al., 2013).

Observaciones: La apariencia general de la valva y sus características son semejantes a $L$. andina. Sin embargo, el contorno de esta especie es más elíptico, el área axial más angosta y el número de areolas (2-3) y la densidad de estrías (16-20 en 10 $\mu \mathrm{m})$ son menores que en los ejemplares observados en nuestro material.

Material observado: TC1: muestras 1, 9, 17, 25, $33,41,49,57,65,73,81,105,121,145,161,129$, 217, 225; PCc2: muestras 7, 9, 10, 11 y 12 .

Luticola cf. elegans (West \& West) Kohler \& Kopalová Fig. 2: G

Kohler et al. (2015).

Valva elíptico-lanceolada con ápices subcapitados. Área axial amplia, recta; área central amplia y transversalmente rectangular. Ramas del rafe rectas, con las fisuras proximales y distales levemente curvadas en el sentido opuesto al estigma. Estrías radiales a lo largo de toda la valva, compuestas por 3-4 areolas.

Dimensiones: eje apical $28 \mu \mathrm{m}(\mathrm{N}=1)$; eje transapical $7 \mu \mathrm{m}(\mathrm{N}=1)$; estrías 18 en $10 \mu \mathrm{m}(\mathrm{N}=1)$.

Observaciones: A pesar de que todas las características del único ejemplar observado son idénticas a las de L. elegans, no podemos afirmar que pertenece a esta especie ya que fue definida como endémica para la Antártida (Kohler et al., 2015). Por esta razón, para lograr una identificación más precisa, resulta imprescindible la observación de más ejemplares.

Material observado: TC1: muestra 25.

Luticola cf. muticopsis (Van Heurck) D.G. Mann Figs. 6: A-C

Levkov et al. (2013): 171: Figs. 127: 20-37; 128: 1-5; 135: 4-5; 142: 17-32

Valvas lineales, con el margen del lado del estigma recto y convexo en lado opuesto. Ápices capitados, anchos. Área axial amplia, lineal a lanceolada, levemente expandida cerca del área central; área central amplia, asimétrica, en forma de cuña. Estigma ubicado más próximo al centro de la valva que al margen, con la abertura externa circular. Las ramas del rafe son rectas o levemente curvadas. Las fisuras proximales externas se curvan en sentido opuesto al estigma; las distales son rectas y no llegan hasta el manto. Estrías transapicales radiales a lo largo de toda la valva, notablemente areoladas y compuestas por 2-4 areolas redondeadas hasta alargadas transversalmente.

Dimensiones: eje apical 16,5-22,0 $\mu \mathrm{m}(\mathrm{N}=8)$, eje transapical 5,5-8,0 $\mu \mathrm{m}(\mathrm{N}=8)$; estrías $19-20$ en 10 $\mu \mathrm{m}(\mathrm{N}=7)$.

Observaciones: El material observado difiere ligeramente de la descripción que figura en Levkov et al. (2013): L. muticopsis tiene un número algo menor de areolas por estría (2-3) y de estrías en $10 \mu \mathrm{m}(15-18)$ y el estigma esta localizado a mitad de camino entre el centro y el margen de la valva. Como sucede con otras especies del mismo género encontradas en este testigo, las citas existentes para esta son solo para la región antártica y sub-antártica, según Luchini \& Verona (1972) y se trataría de una especie endémica de Antártida, siendo su límite norte de distribución las islas Georgias del Sur (Fukushima, H. 1970 en Luchini \& Verona op. cit.). Material observado: TC1: muestras 1, 25 y 129.

\section{Conclusiones}

La combinación entre un mayor detalle de los caracteres morfológicos observados -vinculados al uso extendido del MEB- y el fuerte peso que ha adquirido en los últimos tiempos el criterio biogeográfico para la descripción de nuevas especies han conducido a un incremento de las revisiones taxonómicas publicadas en los últimos años. Este estudio pone de manifiesto la dinámica situación de la taxonomía de diatomeas en la actualidad y la necesidad de realizar este tipo de estudios en regiones geográficas más amplias, con énfasis en aumentar el conocimiento de este grupo de algas en regiones hasta ahora pobremente exploradas. En este trabajo este criterio ha sido utilizado como un carácter más a la hora de distinguir los taxones altoandinos de otros con morfologías semejantes, pero conocidos como endémicos de otros ambientes extremos.

En este trabajo, además, damos a conocer los primeros resultados del análisis de la diversidad del genero Luticola en las inmediaciones de la localidad 
de Abdón Castro Tolay, donde hasta el momento hemos identificado diez especies, de las cuales dos son nuevas (L. punae y $L$. juliae), una se cita por primera vez para Sudamérica y dos representan nuevos registros para Argentina y, además, describimos dos morfotipos de Luticola mollis.

El material recuperado de la localidad de Abdón Castro Tolay no ha sido exhaustivamente estudiado y aún restan por identificar varios especímenes que requieren más observaciones con MO y MEB para su correcta asignación taxonómica. Este material será objeto de posteriores publicaciones.

Consideramos que este trabajo aporta al conocimiento de la diversidad y distribución de Luticola en la Puna Argentina, en el marco del estudio de la biodiversidad de diatomeas en ecosistemas acuáticos de altura que este grupo de trabajo viene desarrollando en las últimas décadas.

\section{Agradecimientos}

Al grupo de investigación de $\mathrm{H}$. Yaccobaccio por su colaboración durante los trabajos de campo. A B. Oxman y J. Kulemeyer por recuperar el testigo TC1. A Z. Levkov y M. Lujan García por sus comentarios vinculados a aspectos taxonómicos de algunas de las especies mencionadas. Este trabajo fue financiado por el PICT 2014-2863.

\section{Bibliografía}

BATTARBEE, R. W. 1986. Diatom analysis. In: BERLUND, B. E. (ed.), Handbook of Holocene Paleoecology and Palaeohydrology, pp. 527-570. J. Wiley \& Sons Ltd., New York.

ESPOSITO, R. M. M., S. A. SPAULdING, D. M. MCKNIGHT, B. VAN DE VIJVER, K. KOPALOVÁ, D. LUBINSKI \& T. WHITTAKER. 2008. Inland diatoms from the McMurdo Dry Valleys and James Ross Island, Antarctica. Botany 86: 1378-1392.

GARREAUD, R. D., M. VUILLE \& A. C. CLEMENT. 2003. The climate of the Altiplano: observed current conditions and mechanisms of past changes. Palaeogeogr. Palaeoclimatol. Palaeoecol. 194: 5-22.

GARREAUD, R. D., M. VUILLE, R. COMPAGNUCCI \& J. MARENGO. 2009. Present-day South American climate. Palaeogeogr. Palaeoclimatol. Palaeoecol. 281: 180-195.
GLUSHCHENKO, A. M. \& M. S. KULIKOVSKIY. 2015. Species of the genus Luticola (Bacillariphiyceae) in waterbodies of Laos and Vietnam. Bot. J. 100: 799804.

HAMMER, Ø., D. A. T. HARPER \& P. D., RYAN. 2001. PAST: Paleontological statistics software package for education and data analysis. Palaeontologia Electronica 4: 9pp. http://palaeo-electronica. org/2001_1/past/issue1_01.htm

KOHLER, T. J., K. KOPALOVÁ, B. VAN DE VIJVER \& J. P. KOCIOLEK. 2015. The genus Luticola D.G. Mann (Bacillariophyta) from the McMurdo Sound Region, Antarctica, with the description of four new species. Phytotaxa 208: 103-134.

KOPALOVÁ, K., J. ELSTER, L. NEDBALOVÁ \& B. VAN DE VIJVER. 2009. Three new terrestrial diatom species from seepage areas on James Ross Island (Antarctic Peninsula Region). Diatom Res. 24: $113-122$.

KRAMMER, K. \& H. LANGE-BERTALOT. 1986. Bacillariophyceae 1. Teil: Naviculaceae. In: ETTL, H., J. GERLOFF, H. HEYNIG \& D. MOLLENHAUER (eds.), Süßwasserflora von Mitteleuropa 2, pp. 876. G. Fischer, Jena.

KRAMMER, K. \& H. LANGE-BERTALOT. 1988. Bacillariophyceae 2. Teil: Bacillariaceae, Epithemiaceae, Surirellaceae. In: ETTL, H., J. GERLOFF, H. HEYNIG \& D. MOLLENHAUER (eds.). Süßwasserflora von Mitteleuropa 2, pp. 596. G. Fischer, Jena.

KRAMMER, K. \& H. LANGE-BERTALOT. 1991a. Bacillariophyceae 3. Teil: Centrales, Fragilariaceae, Eunotiaceae. In: ETTL, H., J. GERLOFF, H. HEYNIG \& D. MOLLENHAUER (eds.). Süßwasserflora von Mitteleuropa 2/3, pp. 576. G. Fischer, Jena.

KRAMMER, K. \& H. LANGE-BERTALOT. 1991b. Bacillariophyceae. 4. Teil: Achnanthaceae Kritische Ergänzungen zu Navicula (Lineolatae) und Gomphonema. In: ETTL, H., J. GERLOFF, H. HEYNIG \& D. MOLLENHAUER (eds.). Süßwasserflora von Mitteleuropa 4, pp. 437. G. Fischer, Jena.

LEVKOV, Z., D. METZELTIN \& A. PAVLOV. 2013. Luticola and Luticopsis. Diatoms of Europe Volumen 7. H. LANGE-BERTALOT (ed). Koeltz Scientific Books.

LUCHINI, L. \& C. A. VERONA. 1972. Catálogo de las diatomeas argentinas. I. Diatomeas de aguas continentales (incluido el Sector Antártico). Comisión de Investigaciones Científicas de la Provincia de Buenos Aires. Monografías, 2, La Plata.

MAIDANA, N. I., M. S., VIGNA \& V. MASCITTI. 1998. Ficoflora de la laguna de Pozuelos (Jujuy, Argentina) I: Bacillariophyceae. Bol. Soc. Argent. 


\section{S. Bustos et al. - Diversidad del género Luticola en la Puna Jujeña}

Bot. 33: 171-179.

MAIDANA, N. I. \& C. SEELIGMANN. 2006. Diatomeas (Bacillariophyceae) de ambientes acuáticos de altura de la Provincia de Catamarca, Argentina II. Bol. Soc. Argent. Bot. 41: 1-13.

MAIDANA, N. I., C. SEELIGMAN \& M. R. MORALES. 2009. Bacillariophyceae del Complejo Lagunar Vilama (Jujuy, Argentina). Bol. Soc. Argent. Bot. 44: 257-271.

MAIDANA, N. I., C. SEELIGMAN \& M. R. MORALES. 2011. El género Navicula sensu stricto (Bacillariophyceae) en humedales de altura de Jujuy, Argentina. Bol. Soc. Argent. Bot. 46: 13-29.

METZELTIN, D. \& H. LANGE-BERTALOT. 2007. Tropical Diatoms of South America II. Icongrafphia Diatomológica Vol.18. A.R.G. Gantner Verlag K. G. Köningstein.

METZELTIN, D., H. LANGE-BERTALOT \& F. GARCÍA-RODRIGUEZ. 2005. Diatoms of Uruguay. Icongrafphia Diatomológica Vol.15. A.R.G. Gantner Verlag K. G. Köningstein.

MORALES, M. R., P. TCHILINGUIRIAN, M. PIROLA, S. BUSTOS \& H. D. YACOBACCIO. 2014. New Holocene paleoenvironmental records from the Dry Puna, Northwestern Argentina. Book of Abstracts of the 4th Southern Deserts Conference. Mendoza, Argentina, pp. 81.

MORALES, M. R., P. TCHILINGUIRIAN, M. PIROLA, S. BUSTOS, B. I. OXMAN, L. PUPO \& M. L. GARCIA. 2015. Nuevos registros paleoambientales de la puna jujeña: hacia un modelo sobre la dinámica y diversidad de los hábitats holocénicos. Libro de resúmenes de las Primeras Jornadas sobre Altiplano Sur. Jujuy, Argentina, pp. 71.

PIROLA, M., S. BUSTOS \& M. R. MORALES. 2015. Preliminary paleoenvironmental interpretation of the 11,500- 4000 BP span from Cruces core 1, dry puna of Jujuy, Argentina: The diatom and C (TOC and TIC) content records. Book of Abstracts of the 4th Southern Deserts Conference. Mendoza, Argentina, pp. 87.

ROUND, F. E., R. M. CRAWFORD \& D. G. MANN. 1990. The Diatoms. Biology and Morphology of the genera. Cambridge University Press, Cambridge.

RUMRICH, U., H. LANGE-BERTALOT \& M. RUMRICH. 2000. Diatomeen der Anden von Venezuela bis Patagonien/Tierra del Fuego. In: H. LANGE-BERTALOT (ed.), Iconogr. Diatolmol 9, pp. 1- 673. A.R.G. Gantner Verlag K. G., Ruggell.
SEELIGMANN, C. \& N. I. MAIDANA. 2003. Diatomeas (Bacillariophyceae) en ambientes de altura de la provincia de Catamarca (Argentina). Bol. Soc. Argent. Bot. 38: 39-50.

SEELIGMAN, C. T., N. I. MAIDANA \& M. R. MORALES. 2008. Diatomeas (Bacillariophyceae) de Humedales de Altura de La Provincia de JujuyArgentina. Bol. Soc. Argent. Bot. 43: 1-17.

VAN DE VIJVER, B., Y. FRENOT \& L. BEYENS. 2002a. Freshwater diatoms from Ile de la Possession (Crozet Archipelago, sub-Antarctica) Biblioth. Diatomol. 46, pp. 41. J. Cramer, Stuttgart.

VAN DE VIJVER, B., P. LEDEGANCK, \& L. BEYENS. 2002b. Soil diatom communities from Ile de la Possession (Crozet, sub-Antarctica). Polar biol. 25: 721-729.

VAN DE VIJVER, B., H. VAN DAM \& L. BEYENS. 2006. Luticola higleri sp. nov., a new diatom species from King George Island (South Shetland Islands, Antarctica). Nova Hedwigia 82: 69-79.

VAN DE VIJVER, B. \& G. MATALONI. 2008. New and interesting species in the genus Luticola D.G. Mann (Bacillariophyta) from Deception Island (South Shetland Islands). Phycologia 47: 451-467.

VAN DE VIJVER, B., R. ZIDAROVA \& M. DE HAAN. 2011. Four new Luticola taxa (Bacillariophyta) from the South Shetland Islands and James Ross Island (Maritime Antarctic Region). Nova Hedwigia 92: 137-158.

ZHOU, J. \& K. M. LAU. 1998. Does a monsoon climate exist over South America? J. Climate 11: 1020-1040.

ZIDAROVA, R., Z. LEVKOV. \&, B. VAN DE VIJVER. 2014. Four new Luticola taxa (Bacillariophyta) from Maritime Antarctica. Phytotaxa 170: 155-168.

Recibido el 17 de mayo de 2016, aceptado el 24 de agosto de 2016. 
